\title{
NUMERICAL BOUNDARY CONDITIONS FOR SOLAR MAGNETOHYDRODYNAMIC FLOWS USING THE METHOD OF CHARACTERISTICS
}

\author{
S. T. WU, A. H. WANG and W. P.GUO \\ The University of Alabama in Huntsville, Huntsville, AL 35899 USA
}

\begin{abstract}
We discuss the self-consistent time-dependent numerical boundary conditions on the basis of theory of characteristics for magnetohydrodynamics (MHD) simulations of solar plasma flows. The importance of using self-consistent boundary conditions is demonstrated by using an example of modeling coronal dynamic structures. This example demonstrates that the self-consistent boundary conditions assure the correctness of the numerical solutions. Otherwise, erroneous numerical solutions will appear.
\end{abstract}

Key words: MHD Simulation - MHD Characteristics - Corona

\section{Introduction}

From recent observational and theoretical studies, we have recognized that the evolution of the solar magnetic field and its interaction with plasma flows are the major physical processes which are responsible for the global solar atmosphere dynamics. These physical processes are best described by the theory of magnetohydrodynamics (MHD) (Parker 1979; Priest 1981). The mathematical model for the atmospheric dynamics in the context of MHD theory is highly nonlinear; it is almost impossible to seek meaningful analytical solutions for realistic physical problems. Therefore, we need to utilize appropriate numerical methods to seek approximate solutions which are known as numerical simulations. It is well- known that the treatment of appropriate boundary conditions is the key to the success of MHD simulation of a realistic physical situation. To proceed otherwise will lead to an erroneous physical solution and misinterpretation of the observed physical features. One of the theoretical methods for treating the boundary conditions is to apply the method of characteristics. Nakagawa (1981) first brought this theory to our attention but without specific numerical examples. Wu et al $(1983,1984,1986)$ employed this formulation to treat wave and mass transport in the solar atmosphere and flare energy build-up due to photospheric shear. In particular, $\mathrm{Hu}$ and $\mathrm{Wu}$ (1984) developed a numerical algorithm named "Fully- Implicit-Continuous-Eulerian (FICE) scheme to implement the method of characteristics for the treatment of boundary conditions for the MHD numerical simulation of initial-boundary value problems. To illustrate this scheme, Nakagawa, $\mathrm{Hu}$ and $\mathrm{Wu}$ (1987) presented a study of the evolution of magnetic arches in the solar atmosphere. Through these studies, we found the importance of the method of characteristics for boundary 
conditions in the numerical MHD simulation. In particular, when the flow is in the subsonic and sub-Alfvenic regimes, the solution in the computational domain will effect the properties on the boundary and vice-versa. This means that there is a coupling between the parameters at the boundary and properties (solution) in the computational domain. The proper mathematical model governing this coupling relationship involves the compatibility equations derived from the theory of characteristics as given by $\mathrm{Wu}$ and Wang (1987) and Nakagawa, $\mathrm{Hu}$ and $\mathrm{Wu}$ (1987). Moreover, Wu and Wang (1987) presented a complete set compatibility equations to govern the boundary conditions according to the flow regimes. It has been demonstrated that conventional fixed boundary conditions will lead to erroneous solutions (Sun et al, 1995). However, it is our opinion that the conventional fixed boundary will be suitable for laboratory conditions. We know that laboratory conditions are controlled conditions; therefore, the boundary conditions determine the solution in the domain of interest. On the contrary, there is strong coupling in astrophysical and space physical problems when the flow in the subsonic and sub-Alfvenic regime exists between the solution in the domain of interest (which represents the observables) and the boundary conditions for which we have no way of knowing a priori. Thus, the method of characteristics boundary conditions must be used. In this paper we shall further illustrate the importance of this type of boundary conditions by using a two-dimensional, time-dependent MHD model for coronal structures. A brief discussion of the fundamental idea of the method of characteristics is given in Section 2. Numerical results are presented in Section 3 , and concluding remarks are included in Section 4.

\section{Fundamental Idea of Method of Characteristics}

We refer to the work of $\mathrm{Hu}$ and $\mathrm{Wu}$ (1984); Wu and Wang, (1987); Nakagawa, $\mathrm{Hu}$ and $\mathrm{Wu}(1987)$ and most recently, Sun, Wu and Dryer (1995) for the detailed treatment of the method of characteristics. Here we shall briefly summarize some of the important ideas.

Let us consider the simplest partial differential equation system of a function $f(x, t)$ of two independent variables $x$ and $t$ such as:

$$
\frac{\partial f}{\partial t}+u \frac{\partial f}{\partial x}=g
$$

Equation (1) can be written as a total differential equation of the form

$$
\frac{d f}{d s}=g
$$


This equation provides the definition of the directional derivative in the $(x, t)$ plane along a curve $\mathrm{s}(\mathrm{x}, \mathrm{t})$ to be:

$$
\frac{d f}{d s}=\frac{\partial f}{\partial t} \frac{\partial t}{\partial s}+\frac{\partial f}{\partial x} \frac{\partial x}{\partial s}
$$

where the direction of the curve $s(x, t)$ is

$$
\frac{d x}{d t}=\frac{\frac{\partial s}{\partial t}}{\frac{\partial s}{\partial x}}=u .
$$

Thus, the solution of Eq. (1) can be obtained by integrating Eq. (2) along the curve $s(x, t)$ for both linear and nonlinear cases of $u$ and $g$ being general functions of $\mathrm{x}$ and $\mathrm{t}$ in the form

$$
f(s)=f(o)+\int_{0}^{s} g d s
$$

with $f(o)$ being the values of $f(x, t)$ at $s=o$.

This is the fundamental idea of the method of characteristics to obtain the solutions of nonlinear hyperbolic partial differential equations. The curves along which total differential equations can be derived are called the characteristics, while the resultant equations are called the compatibility equations. In practical MHD problems, various physical variables correspond to the function $f(x, t)$ and the compatibility equations can be obtained from combinations of physical variables along a number of characteristics in a one spatial dimensional problem (Akiezer et al. 1975) or a more general two or three-dimensional problem. We refer readers to the recent works (Nakagawa, 1981; Hu and $\mathrm{Wu}, 1984$; Nakagawa, $\mathrm{Hu}$ and $\mathrm{Wu}, 1987$; $\mathrm{Wu}$ and Wang, 1987; and Sun, Wu and Dryer, 1995) for a more extensive and complete development.

\section{Numerical Examples}

To illustrate the effects of the boundary conditions on the solution, we have chosen a two-dimensional, time-dependent MHD model in spherical coordinates for a coronal streamer structure. The governing equations and method of calculation are given by Wang et al (1993). By setting up all the physical conditions to be identical, we used three different types of boundary conditions for this test. These three different types of boundary conditions are: (i) all fixed values (i.e., density, temperature, velocity and magnetic field strength) on the lower boundary; (ii) time-dependent boundary condtions using method of characteristics; and (iii) time-dependent boundary conditions using a numerical procedure (i.e., linear extrapolation). These three 
types of boundary conditions represent two catagories; Case 1 is the conventional way to specify the boundary conditions. This type of boundary condition is suitable for those problems with controlled conditions on the boundary (i.e., laboratory experiments). Cases 2 and 3 are for the cases in which the properties on the boundary must vary to satisfy the realistic physical conditions. To implement the time-dependent boundary conditions, we have used two techniques; (a) method of characteristics (Wu and Wang, 1987) for Case 2 and (b) numerical procedure (i.e., linear extraplation) for Case 3. The results for density and radial velocity distribution are shown in Figure 1 which immediately demonstrate that Case 1 showed development of erroneous solution when compared with Case 2. In fact, we tried unsuccessfully to obtain a solution for Case 3 .

\section{Concluding Remarks}

In this study we presented the fundamental idea of the method of characteristics as the numerical boundary conditions for the numerical simulation of solar atmospheric dynamics. The importance of this type of self-consistent boundary conditions was outlined. Presently, a number of authors (Wu et al. 1983; 1984; 1995; Wang et al, 1993) have applied this procedure to a number of dynamical features such as coronal streamers, initiation of CMEs and flare energy buildup. Most recently Linker et al(1994) and Linker and Mikic (1995) also emphasized the importance of the self-consistent method of characteristic boundary conditions that we have developed. Finally, we conclude that the application of self-consistent boundary conditions are crucial to obtain correct results for numerical MHD simulations.

\section{Acknowledgements}

This work is supported by an NSF grant ATM-9251673 and a NASA/MSFC grant NCC8-65. We acknowledge Dr. M. Dryer for reading the manuscript and giving valuable suggestions.

\section{References}

Akhizer, A. J., Polovin, R.V., Sitenko, A. G., and Stepanov, K. N.: 1975, Plasma Electrodynamics, Pergamon Press, 1

$\mathrm{Hu}, \mathrm{Y} . \mathrm{Q}$. and $\mathrm{Wu}, \mathrm{S}$. T.: 1984, J. Comp. Phys, 55, 33

Mikic, Z. and Linker, J. A.: 1995, Astrophysical Journal 438, 645

Linker, J. A., Mikic, Z. and Schnack, D. D.: 1994, Solar Dynamic Phenomena and Solar Wind Consequences, SOHO III Workshop, ESA Report SP-373, p. 249

Nakagawa, Y.: 1981, Astrophysical Journal 247, 719 
DENSITY
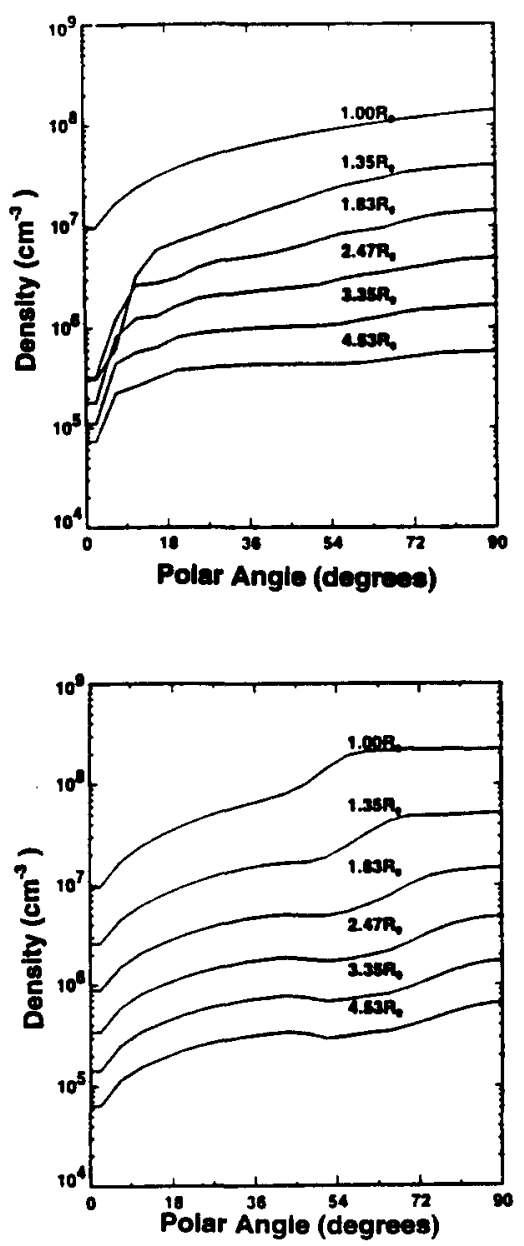

VELOCITY

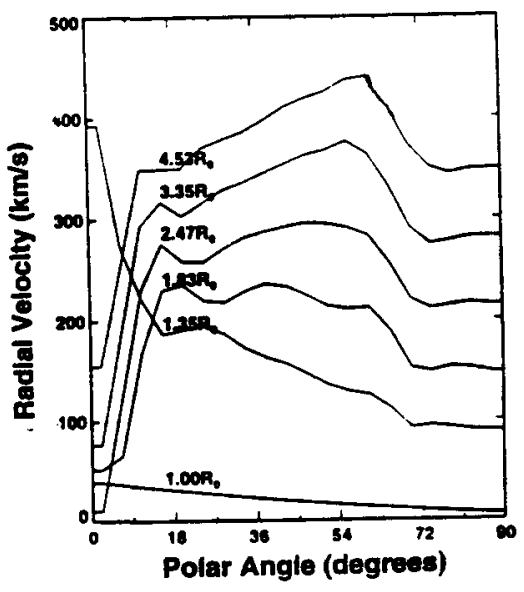

CASE 1

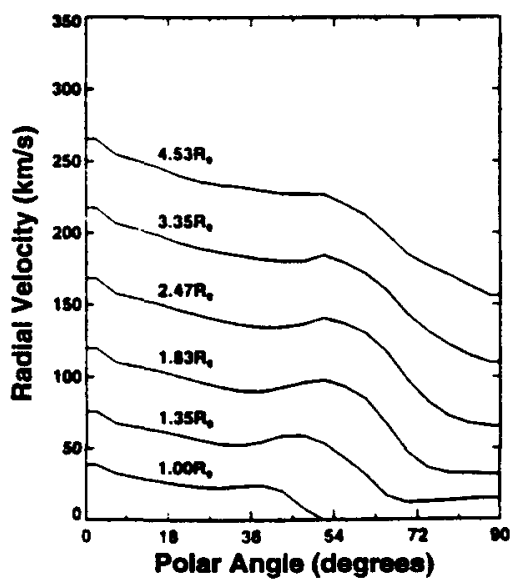

CASE 2

Fig. 1. The results for density and radial velocity distribution for Case 1 and Case 2 .

Nakagawa, Y., Hu, Y. Q., and Wu, S. T.: 1987, aaa 179, 354

Parker, E. N.: 1979, Cosmical Magnetic Fields, Clarendon Press, Oxford

Priest, E. R.: 1981, Solar Flare Magnetohydrodynamics, Gordon and Breach Science Publishers, New York

Sun, M. T., Wu, S. T., and Dryer, M.: 1995, J. Comp. Phys, (in press)

Wang, A. H., Wu, S. T., Suess, S. T., and Poletto, G.: 1993, sph 147, 55

Wu, S. T., Hu, Y. Q., Nakagawa, Y., and Tandberg-Hanssen, E: 1983, Astrophysical Journal 266, 866

Wu, S. T., Hu, Y. Q., Krall, K. R., Hagyard, M. J. and Smith, Jr., J. B.: 1984, sph 90, 117

Wu, S. T., Nakagawa Y., and Tandberg-Hanssen, E: 1986, Astrophysical Journal 306, 751 Wu, S. T. and Wang J. F.:1987, J. Comp. Methods in Applied Mech. and Eng'g, 64, 267

Wu, S. T., Guo, W. P., and Wang, J. F.: 1995, sph, in press. 